\title{
Un nuevo profesional para una nueva sociedad. Respues- tas desde la educación médica: la formación basada en competencias.
}

New professionals for a new society. Responses from a medical education perspective: competency-based medical education.

\author{
Jesús Morán-Barrios ${ }^{a}$. \\ ${ }^{a}$ Jefe de Estudios de Formación Sanitaria Especializada. Presidente de la Sociedad de Educación Médica \\ de Euskadi. Unidad de Docencia Médica. Hospital Universitario Cruces. Barakaldo. Vizcaya, España.
}

Correspondencia: Jesús Morán-Barrios (jesusmanuel.moranbarrios@osakidetza.net)

RESUMEN: La formación de profesionales de Ciencias de la Salud exige una reflexión permanente para dar respuesta a una sociedad en continua transición, máxime cuando se viven momentos sociales y económicos tan delicados como el actual que influyen directamente en el mundo sanitario. En un mundo globalizado, en continuo cambio, lleno de incertidumbres, donde la movilidad de profesionales entre países es creciente, se precisan profesionales con nuevas competencias para que sean capaces de adaptarse con flexibilidad a los nuevos tiempos, nuevos roles de docentes y discentes, así como nuevas metodologías de educación y evaluación. La Formación basada en Competencias (FBC) es el camino a seguir para dar una respuesta a los retos socio-sanitarios actuales.

En este artículo desarrollamos los fundamentos de la FBC, exponemos algunos de los modelos más aceptados a nivel internacional, describimos cual debe ser el perfil básico de un profesional en Ciencias de la Salud hoy para el mañana, y mostramos la experiencia puesta en marcha por la Unidad de Docencia Médica del Hospital Universitario Cruces de implementar la FBC en el programa de especialidades.

PALABRAS CLAVE: Formación Sanitaria Especializada. Formación Médica basada en Competencias.
ABSTRACT: Ongoing reflection on the training of Health Sciences professionals is essential to respond to changes in society. This is particularly important in testing times, such as those of the current economic and social conditions, which have a direct impact on health systems. In the era of globalisation, with the world in constant change, full of uncertainties, and increasing movement of professionals between countries, we need: professionals with new competencies, flexible in adapting to the new times; and new teaching and learning roles, as well as new educational and assessment methods. Competency-based medical education (CBME) is the best approach to respond to the current social and healthcare challenges.

In this paper, we outline the principles of CBME, present some of the models most widely adopted internationally, describe the framework of basic competencies that Health Science professionals need today to meet the challenges of tomorrow, and report our experience of the introduction of CBME in specialty training programmes at the Medical Education Unit of Cruces University Hospital.

KEY WORDS: Postgraduate Medical Education, Competency-Based Medical Education. 


\section{Introducción}

Ya no nos vale con afirmar, "el sistema MIR es bueno", hay que demostrarlo y contrastarlo con otros sistemas formativos. Para ello, es crucial definir qué perfil profesional queremos formar adaptado a las necesidades sociales y del sistema sanitario y medir resultados educativos. Debemos preguntarnos: $¿$ estamos formando el perfil profesional que la sociedad y el sistema sanitario necesita?, ¿qué peso deben de tener los médicos generalistas respecto a los especialistas en los diferentes modelos sanitarios?, ¿y el de otras profesiones sanitarias, como enfermería, auxiliares, asistentes sociales o los educadores sanitarios?, ¿cuál debe ser el papel de las instituciones educativas en los diferentes entornos sociales?, ¿se educa al médico para trabajar multidisciplinarmente?, ¿cómo es la coordinación de las diferentes instituciones educativas en ciencias de la salud con los sistemas de salud?, ¿y entre especialistas de diferentes disciplinas?.

En España, la Formación Sanitaria Especializada precisaba de un urgente impulso, ya que, desde 1984 no se habían modificado sus normas básicas de funcionamiento. El reciente marco regulatorio (Real Decreto 183/2008) y su desarrollo por las CCAA, nos permitirá avanzar y desarrollar aquellas iniciativas e innovaciones que en Educación en Ciencias de la Salud son imprescindibles implantar en los centros sanitarios, para responder a una nueva sociedad y adecuarnos al contexto educativo y de práctica profesional internacional. Ello implica que los centros sanitarios del Sistema Nacional de Salud, consoliden los recursos necesarios para la formación (tiempo para la gestión de la formación, espacios educativos o recursos humanos con competencias en educación, entre otros) y desarrollen indicadores contrastables de la gestión de su responsabilidad educativa. Las instituciones sanitarias españolas (centros y servicios asistenciales), tienen que desarrollar sus propias experiencias dentro del marco general que proporciona la legislación vigente. Estas instituciones deben ser conscientes del compromiso adquirido con la sociedad a través de la acreditación docente, precisan consolidar su organización educativa y a los distintos agentes formadores (Jefes de Estudio y Tutores), e incorporar otras figuras docentes como psicólogos, sociólogos o pedagogos, ejerciendo un liderazgo efectivo para el completo desarrollo de los programas formativos. Es preciso, además, que las Comunidades Autónomas desarrollen sus propias normativas en Formación Sanitaria Especializada. Todo ello, para responder a la sociedad con un profesional competente, capaz de moverse en cualquier contexto sanitario, con flexibilidad y autonomía. 
PARA EL DEBATE

\section{Globalización y educación.}

El profesional, el modelo de sistema sanitario (su financiación y gestión) y el ciudadano, son tres elementos que no siempre están en armonía. Desde el punto de vista del ciudadano, el concepto de salud y enfermedad es una percepción en la que influyen factores culturales, económicos, políticos, o mediáticos, entre otros. La frontera entre salud y enfermedad se hace aún más débil en lo que a la salud mental se refiere, existiendo, a veces, distorsiones influenciadas por la industria farmacéutica y biotecnológica, donde sus intereses y las necesidades de salud no siempre confluyen, habiéndose dado circunstancias que han supuesto penalizaciones por parte de las autoridades reguladoras (1).

De aquí que surjan disfunciones entre ciudadanos, sistemas sanitarios y profesionales. Y todo ello hay que incluirlo en el actual mundo globalizado con un reparto de riqueza desigual, con presiones derivadas del enorme desarrollo científico y tecnológico y de las crecientes necesidades humanas, donde, además, la movilidad de personas y las migraciones siguen una curva creciente imparable, lo que genera nuevas distorsiones. Sociedades que eran estables están dejándolo de ser, y la medicina no es ajena a ello.

Hasta pasados los primeros años del siglo XXI, no éramos conscientes del impacto que sobre nuestras vidas podían tener decisiones tomadas por otros a los que desconocemos, y cuyas consecuencias (la actual crisis económica y de valores) están siendo devastadoras para nuestra sociedad y afectarán de modo permanente a los ámbitos políticos, socio-culturales y morales de nuestras sociedades. Estamos viendo la otra cara de la moneda de la globalización, un fenómeno de interdependencia económica que se está viviendo intensamente desde los años 90 y que ha dado lugar a las políticas neoliberales que conocemos. La globalización está en la economía, pero también tiene sus efectos en la política, la ciencia, la cultura, la educación y la sanidad (2).

Los modelos educativos no son ajenos a las corrientes socioeconómicas ni a los sistemas de atención sanitaria, debiendo estar al servicio de estos. Los sistemas sanitarios centrados en el hospital (más tecnológicos y focalizados en la enfermedad), o los centrados en la atención primaria (el individuo y la comunidad), influyen en el sistema educativo y en el perfil profesional, y viceversa. Sin embargo, en los últimos veinte años, ha emergido un elemento muy poderoso que condiciona los sistemas educativos y sanitarios a través del dominio que ejerce sobre los ciudadanos. Este elemento es el "mercado" y los medios de comunicación ligados al mismo. Los fuertes intereses económicos del mercado influyen directamente sobre los deseos y percepciones en salud de los ciudadanos y sobre las decisiones de los estados en materia sanitaria. Es decir, como ya hemos apuntado más arriba, en el concepto y percepción de salud y enfermedad influyen múltiples factores externos a la persona. 
La formación de profesionales ha de plantearse cambios si realmente quiere contribuir a paliar algunos de los inconvenientes derivados de la globalización. El compromiso de las instituciones formativas ha de ser el de formar profesionales por y para la comunidad; con la posesión de unos valores comunitarios que expresen cierta solidaridad y que desarrollen la empatía, para ser capaces de ponerse en la piel y en el sentir del otro. Este compromiso no se tiene que quedar sólo en documentos, sino que ha de manifestarse a través de los programas de formación y de las actuaciones de los propios docentes. Tal compromiso se ha de centrar en una formación que potencie, entre otros aspectos: la convivencia entre las distintas culturas, el aprendizaje a lo largo de la vida, la autonomía y responsabilidad personal y profesional, una visión universalista, y el pensamiento crítico, creativo y solidario (2). En definitiva, la formación debe de hacer énfasis en los principios del "profesionalismo" o profesionalidad (3) (tabla 1).

Tabla 1.

El Profesionalismo en el nuevo milenio.

Las responsabilidades profesionales implican un compromiso con:

1. La competencia: aprendizaje a lo largo de toda la vida profesional.

2. La honestidad con los pacientes.

3. La confidencialidad.

4. El mantenimiento de unas relaciones apropiadas con los pacientes.

5. La mejora en la calidad de los cuidados.

6. La mejora en el acceso a los cuidados.

7. La justa distribución de recursos finitos.

8. El conocimiento científico: integridad en el uso del conocimiento y la tecnología.

9. El mantenimiento de la confianza en la gestión del conflicto de intereses.

10. Las responsabilidades profesionales: trabajo colaborativo, autorregulación.

Los fines de la medicina y el modelo de educación como respuesta a las necesidades de la sociedad y del sistema sanitario.

The Hasting Center, el prestigioso centro estadounidense de investigación en bioética, editó en 1996 un documento fundamental titulado Los Fines de la Medici$n a$ (4) elaborado por un numeroso equipo de expertos internacionales de diferentes nacionalidades y culturas (Alemania, Chile, China, Dinamarca, España, Estados Unidos, Holanda, Hungría, Indonesia, Italia, Reino Unido, República Checa, República Eslovaca y Suecia), que a su vez trabajaron con expertos de sus propios países. Las variables de partida para definir dichos fines fueron tres: 1) El progreso de la ciencia y la biotecnología, 2) el aumento de las necesidades humanas y 3) la escasez de recursos para satisfacerlas. Los cuatro fines acordados por consenso 
PARA EL DEBATE

fueron: 1) La prevención de enfermedades y lesiones y la promoción y la conservación de la salud; 2) el alivio del dolor y el sufrimiento causados por males; 3) la atención y la curación de los enfermos y los cuidados a los incurables; y 4) la evitación de la muerte prematura y la búsqueda de una muerte tranquila.

Por otra parte, el informe de la Comisión Global Independiente "Education of Health Professionals for the $21^{\text {st }}$ Century" formada por expertos educadores de 18 países de diferentes culturas (5), (proyecto financiado por Bill \& Melinda Gates Foundation, Rockefeller Foundation, y China Medical Board), concluye, que, a comienzos del siglo XXI persisten desigualdades en la atención a la salud entre países y dentro de ellos. Se avecinan grandes retos como nuevos riesgos infecciosos, medioambientales y rápidas transiciones demográficas y epidemiológicas que amenazan la seguridad de la salud de todos. Los sistemas de salud del mundo tratan de mantener el nivel requerido haciéndose más complejos y costosos. Además, la educación de los profesionales no va paralela a esos retos al basarse en curruículos fragmentados y estáticos. Existe una descoordinación entre las competencias que se adquieren en la formación y las necesidades de las personas; un deficiente trabajo en equipo; un enfoque técnico limitado sin una visión holística; encuentros episódicos frente a un cuidado de salud continuo; una orientación hospitalaria frente a la atención primaria; y desbalances cualitativos y cuantitativos del mercado laboral. Algunos de los esfuerzos por resolver estos problemas han chocado contra el "tribalismo" de las profesiones (5).

El informe añade que, "todos los profesionales de la salud en todos los países deberán ser entrenados para movilizar el conocimiento y comprometerse al razonamiento crítico y a una conducta ética, de modo que, se hagan competentes para participar en los sistemas de salud centrados en el paciente y la población como miembros activos de los equipos de salud, que proveen una respuesta local y están conectados globalmente". El propósito fundamental es asegurar la cobertura universal de servicios integrales de alta calidad que son esenciales para mejorar las oportunidades de igualdad en los cuidados de la salud entre los países y dentro de ellos.

Respecto a la Educación, tanto el informe del Hasting Center como el de la Comisión "Education of Health Professionals for the $21^{\text {st }}$ Century", incide en el mismo cambio, que de una formación centrada en la enfermedad se transite a una visión holística y sistémica.

La formación de los médicos está orientada al uso de la tecnología, las industrias farmacéuticas y de equipamiento se dedican a desarrollarla y producirla, y los sistemas sanitarios no piensan más que en su empleo y el modo de financiarla (Los Fines de la Medicina) (4). Es evidente que este modelo sanitario genera una espiral de demanda creciente, sin claros beneficios de salud adicionales, o solo marginales y caros, como destaca la OMS, cuya financiación para los Estados puede hacerse inviable, dejando en manos de otros, aquella atención sanitaria más cara o llamé- 
mosla, de élite, con lo que el principio de justicia social y las obligaciones del Estado para con los ciudadanos comienza a tambalearse.

El mismo informe, subraya que, en las sociedades modernas la docencia de la medicina lleva muchas décadas centrándose en el modelo "diagnóstico y tratamiento". Debido a su éxito en muchos casos y a su simplicidad lógica como método, seguirá siendo un modelo central de gran aceptación en la enseñanza de la medicina. Pero sus carencias son muchas: la distorsión de la relación entre médico y paciente; la incapacidad de aportar una buena formación que sirva para abordar las complejidades tanto médicas como sociales de las enfermedades crónicas y las discapacidades; el descuido de la promoción de la salud y la prevención de las enfermedades; y el plano secundario a que se han relegado las humanidades médicas" (4, pág. 64).

Durante el siglo pasado, dos generaciones de reformas educativas caracterizaron el progreso de le medicina. La primera, a principios del siglo XX, planteó un currículo basado en el método científico. La segunda, hacia la mitad del siglo, introdujo innovaciones mediante un currículo basado en la resolución de problemas (diagnóstico-tratamiento). Se necesita una tercera generación con un currículo basado en sistemas para mejorar el rendimiento de los sistemas de salud mediante la adaptación de las competencias profesionales esenciales a contextos específicos (5)

En una sociedad occidental envejecida, los sistemas sanitarios están cambiando su enfoque hacia modelos menos centrados en el hospital, para reorientarlos hacia la prevención y los cuidados primarios de salud, integrando bajo un mismo objetivo las estructuras de atención primaria y hospitalaria. Numerosos gobiernos estatales y regionales en los países desarrollados, han iniciado estrategias de abordaje del enfermo crónico, con acciones centradas en la atención primaria. Para ello, la formación básica del profesional de hoy, debe de reorientarse de una centrada en la enfermedad (órgano y sistemas) a otra, que sin perder lo positivo de ese modelo (ciencia y biotecnología), ponga su atención en la persona enferma, dentro del complejo social de la familia y la comunidad, y en la práctica de una medicina más participativa. La Declaración de Barcelona del año 2003 incide directamente en esto último (6) (tabla 2). 
PARA EL DEBATE

Tabla 2.

Declaración de Barcelona 2003. Decálogo de demandas de los ciudadanos a los servicios de salud.

1. Información de calidad contrastada respetando la pluralidad de las fuentes.

2. Decisiones centradas en el paciente.

3. Respeto a los valores y a la autonomía del paciente informado.

4. Relación médico-paciente basada en el respeto y en la confianza mutua.

5. Formación y entrenamiento específico en habilidades de comunicación para profesionales.

6. Participación de los pacientes en la determinación de prioridades en la asistencia sanitaria.

7. Democratización formal de las decisiones sanitarias.

8. Reconocimiento de las organizaciones de pacientes como agentes de la política sanitaria.

9. Mejora del conocimiento que tienen los pacientes sobre sus derechos básicos.

10. Garantía de cumplimiento de los derechos básicos de los pacientes

\section{Formación basada en competencias (FBC)}

¿Cómo adelantarse a los acontecimientos en la formación de profesionales y en los modelos de atención sanitaria?. Son numerosas las instituciones y organizaciones de nivel nacional o internacional que han definido el perfil del profesional a formar para dar respuesta a los retos sociales. Algunas de ellas se han quedado en la mera recomendación de expertos, sin establecer lo más importante, un plan de implementación de los modelos y un cambio de cultura en la institución educativa o sanitaria, como lo hizo el proyecto EFPO-Educating Future Physicians for Ontario en 1990, donde, además, la voz de los pacientes fue fundamental para definir el perfil del profesional (7), perfil que fue adoptado en el año 2000 por The Royal College of Physicians and Surgeons of Canada (8) para el conjunto de Canadá.

No es posible establecer sistemas educativos y complejos procesos de evaluación, tanto en el grado como en la formación especializada, si no está previamente definido qué profesional se precisa y para qué sociedad. En el documento El futuro de la profesión médica (9), A. Jovell realiza un riguroso análisis sobre qué profesional es necesario para responder a los retos de las transiciones sociales, las cuales están relacionadas con aspectos demográficos, epidemiológicos, científico-tecnológicos, culturales, éticos y de valores, económicos, laborales, político-jurídicos o de nuevos modelos de organización y gestión sanitaria, así como la influencia de los medios de comunicación o la cultura del consumo. La línea argumental de su análisis es válida en el momento actual, cuando tenemos que abordar una crisis tan dramática en España y en Europa, como la económica y de valores, que está afectando directamente a los derechos humanos. Esto implica un nuevo profesional, donde la solidaridad, el liderazgo y la corresponsabilidad con la sostenibilidad del sistema sanitario público sean valores fundamentales. 
El reto de los médicos de hoy está en saber responder de forma efectiva y eficiente a las necesidades de la sociedad del siglo XXI y a la confianza otorgada por los pacientes. Formar hoy al especialista del mañana es proporcionarle todas las competencias necesarias para afrontar las incertidumbres de la futura práctica clínica, gestionar adecuadamente los futuros cambios sociales y los propios de la profesión médica (campo de actuación de las especialidades y la colaboración entre estas), así como entender y encontrar la posición del médico entre los distintos agentes que le influyen (el estado/gobiernos, las organizaciones/corporaciones sanitarias, la industria farmacéutica y de tecnologías sanitarias, los ciudadanos, y otras profesiones sanitarias) (10).

Se precisan, por tanto, sistemas educativos dinámicos que den una respuesta segura, efectiva, eficiente y de calidad a las necesidades y expectativas de los ciudadanos. En definitiva, se trata de implementar en las instituciones académicas y en las sanitarias, estrategias educativas que garanticen la formación del profesional que la sociedad precisa, centrándolo en la calidad de los cuidados y en la seguridad del paciente.

Sin embargo, los sistemas educativos, excesivamente estáticos, no se adaptan con la rapidez suficiente a unas necesidades sociales que emergen a gran velocidad. Es en ese contexto de transformaciones cada vez más importantes y complejas donde la FBC surge para procurar una mayor adaptación y desarrollo de las personas en el mundo laboral (11) (fig. 1). La FBC se centra en el aprendizaje (discente) y no en la enseñanza (docente), y en resultados, flexibiliza el desarrollo de los programas en función del ritmo de aprendizaje y enfatiza la evaluación formativa y la rendición de cuentas (12).

Figura 1.

Formación basada en competencias (Modificado de Frenk (5))

Modelo tradicional basado en objetivos

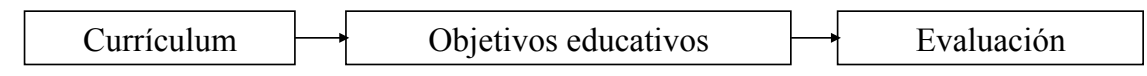

Modelo educativo basado en competencias
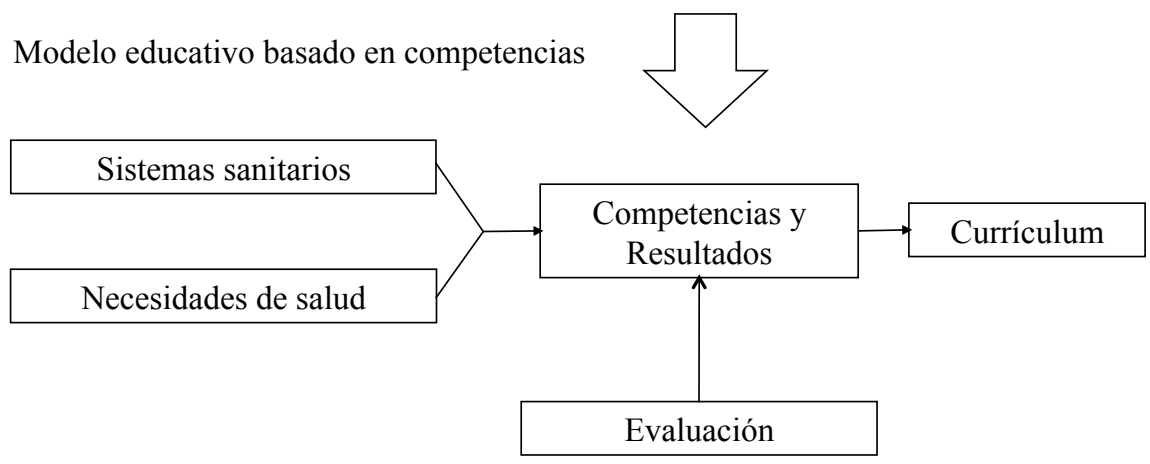
PARA EL DEBATE

Bunk G. P. define las competencias como los comportamientos resultantes de un conjunto de actitudes, habilidades, destrezas, conocimientos y valores que las personas manifiestan para resolver situaciones concretas relacionadas con su vida y su profesión (13). Es decir, una capacidad efectiva para llevar a cabo exitosamente una actividad laboral plenamente identificada; es una capacidad real y demostrada, que se puede evaluar a través de resultados observables, donde se integran los conocimientos (saberes), habilidades y destrezas (saber hacer), actitudes y comportamientos (saber estar), y valores y creencias (saber ser) (14). En definitiva, el desempeño (performance).

Por tanto, "la simple idea de que un profesional competente es aquel que posee los conocimientos y habilidades que le posibilitan desempeñarse con éxito en una profesión específica, ha quedado atrás, siendo sustituida por la comprensión de la competencia profesional como un fenómeno complejo, que expresa las potencialidades de la persona para orientar su actuación en el ejercicio de la profesión, con iniciativa, flexibilidad y autonomía, en escenarios heterogéneos y diversos, a partir de la integración de conocimientos, habilidades, motivos y valores que se expresan en un desempeño profesional eficiente, ético y de compromiso social (aportación personal de Pilar Martínez Clarés, en "Reinventar la formación de médicos especialistas") (2).

El movimiento de las competencias "competency movement" fue impulsado por David McClelland (15), quien en 1973, puso de manifiesto que los test tradicionales de aptitudes académicas, de conocimientos generales y los créditos académicos, no predecían el rendimiento laboral ni el éxito en la vida profesional. Esto le llevó a buscar otras variables, a las que llamó "competencias", que permitiesen una mejor predicción del rendimiento laboral. Para predecir con mayor eficiencia los resultados laborales, era necesario estudiar directamente a las personas en el trabajo. Señaló que más allá de los conocimientos y habilidades, otros factores como los sentimientos, creencias, valores, actitudes y comportamientos pueden augurar o predecir un desempeño altamente satisfactorio en un puesto de trabajo. Hablamos de la empatía, la intuición, la integridad, la percepción de la realidad, el espíritu de comunidad, la autoconfianza, la autocrítica, la flexibilidad, el dominio de la persona. Nació así en Norteamérica, el movimiento de las competencias ("The competency movement"), tanto en el mundo de la psicología de la educación como en el de la psicología industrial y organizacional, cuyo máximo desarrollo ha tenido lugar en la empresa privada.

Lo planteado por McClelland respalda que el conocimiento es una condición necesaria pero no suficiente para un buen desempeño profesional. Hace tiempo que las empresas comprendieron que las competencias de las personas no sólo se deben medir exclusivamente con exámenes de conocimiento o determinados test psicométricos, sino que había que recurrir a otras técnicas que tuviesen en cuenta las experiencias laborales y los comportamientos manifestados en el desempeño en un 
puesto de trabajo observando al trabajador. Estos métodos han sido incorporados a la educación en ciencias de la salud.

En la FBC, evaluación y formación van de la mano y sus aspectos fundamentales son: un sistema de evaluación formativa basado en la observación del comportamiento en el puesto de trabajo y de los resultados del trabajo, una retroalimentación frecuente y de alta calidad (feedback) por parte del evaluador, documentar los resultados de aprendizaje y flexibilizar el programa en función del ritmo de aprendizaje. Por parte de los residentes exige una autoevaluación, un compromiso explícito con su propio proceso formativo y una reflexión continua documentando los puntos fuertes y débiles $(16,17)$. La reflexión es la clave de este proceso de FBC (18) y la evaluación se convierte en el verdadero motor del aprendizaje y del desarrollo profesional. Pasamos de "la evaluación del aprendizaje" a "la evaluación para el aprendizaje", siendo la clave el feedback (19). El tutor es la pieza fundamental de todo este proceso.

No es objeto de este artículo desarrollar los diferentes métodos de evaluación del desempeño y remitimos al lector a otros artículos de tipo general o aplicados a la Psiquiatría (20-24). Recomendamos la consulta de la página oficial del General Medical Council del Reino Unido, donde están publicados los mapas de competencias y métodos de evaluación para Psiquiatría y otras especialidades (http://www.gmc-uk.org/Psychiatry_Blueprint_of_the_Assessment_Programme_ CORE_2009.pdf_30562276.pdf )

\section{Perfil del futuro profesional}

Numerosas organizaciones académicas y sanitarias de distintos países, se adelantaron en la década de los años 90 y principios del 2000, a definir las competencias básicas comunes de sus médicos y especialistas: el General Medical Council del Reino Unido (http://www.gmc-uk.org/education/index.asp), las universidades escocesas con el "Scottish Doctor" (25), Canadá (CanMEDS Roles) (8), Estados Unidos (Outcome Project- Acreditation Council Graduate Medical Education) (26), y a nivel internacional, el Instituto Internacional para la Educación Médica de New York (27) (Tabla 3). Los perfiles profesionales deben responder a los principios del profesionalismo ya citados y es importante enfatizar que los programas educativos incluyan competencias relacionadas con la comunidad, que además de las de salud pública, incluyen las culturales, gestión y liderazgo, defensa y desarrollo de la salud comunitaria, resolución de problemas, creatividad, motivación y autoreflexión (28). 
PARA EL DEBATE

Tabla 3.

Modelos Competenciales de diferentes instituciones, su paralelismo y dominios competenciales adoptados por el Hospital U. Cruces

\begin{tabular}{|c|c|c|c|}
\hline CanMEDs & $\begin{array}{l}\text { Outcome Project } \\
\text { (ACGME) }\end{array}$ & IIEM New York & $\begin{array}{c}\text { Ser Médico/Profesional } \\
\text { en el Hospital U. Cruces } 2008\end{array}$ \\
\hline $\begin{array}{l}\text { 1. Experto Médico } \\
\text { 2. Comunicador } \\
\text { 3. Colaborador } \\
\text { 4. Gestor } \\
\text { 5. Consejero de } \\
\text { salud } \\
\text { 6. Erudito, estu- } \\
\text { dioso } \\
\text { 7. Profesional }\end{array}$ & $\begin{array}{l}\text { 1. Profesionalidad } \\
\text { 2. Habilidades Interper- } \\
\text { sonales y de Comu- } \\
\text { nicación } \\
\text { 3. Conocimiento Médi- } \\
\text { co } \\
\text { 4. Cuidados del Pacien- } \\
\text { te } \\
\text { 5. Práctica basada en el } \\
\text { contexto del Sistema } \\
\text { de Salud } \\
\text { 6. Práctica clínica basa- } \\
\text { da en el aprendizaje } \\
\text { y la mejora }\end{array}$ & $\begin{array}{l}\text { 1. Valores profesionales, } \\
\text { actitudes, comporta- } \\
\text { miento y ética } \\
\text { 2. Habilidades de Co- } \\
\text { municación } \\
\text { 3. Fundamentos científi- } \\
\text { cos de la medicina } \\
\text { 4. Habilidades clínicas. } \\
\text { 5. Salud pública, siste- } \\
\text { mas de Salud } \\
\text { 6. Manejo de la informa- } \\
\text { ción } \\
\text { 7. Análisis critico, au- } \\
\text { toaprendizaje e inves- } \\
\text { tigación }\end{array}$ & $\begin{array}{l}\text { 1. Profesionalidad: valores profesio- } \\
\text { nales, actitudes, comportamiento } \\
\text { y ética } \\
\text { 2. Comunicación } \\
\text { 3. Cuidados del paciente y habilida- } \\
\text { des clínicas } \\
\text { 4. Conocimientos } \\
\text { 5. Práctica basada en el contexto del } \\
\text { Sistema de Salud (Salud Pública } \\
\text { y Sistemas Sanitarios) } \\
\text { 6. Práctica basada en el aprendizaje } \\
\text { y la mejora continua (análisis crí- } \\
\text { tico y autoaprendizaje) } \\
\text { 7. Manejo de la información }\end{array}$ \\
\hline
\end{tabular}

Existe un paralelismo entre los diferentes modelos de competencias, que se adaptan a la cultura y necesidades de cada contexto social. Por ejemplo: el dominio competencial "Salud Pública y Sistemas de Salud" en el modelo del IIEM de Nueva York es superponible al concepto de "Práctica basada en el sistema de salud" (ACGME) o al del modelo canadiense de "gestor y consejero de salud". Unos están dirigidos exclusivamente a la formación de grado y otros a la formación especializada. El modelo del IIEM tiene una amplia aceptación para el grado, y el modelo ACGME o el de CanMEDS para la especialización. Cabe destacar de los modelos del Reino Unido, Tomorrw's Doctor y Good Medical Practice, que la seguridad del paciente es el centro del marco formativo.

En España, la Fundación Educación Médica, a través del consenso de expertos ha editado el documento "El médico del futuro" (29): Un médico que trate enfermos y no enfermedades, con actitud crítica, comunicador y empático, responsable individual y socialmente, que toma buenas decisiones para el paciente y para el sistema, líder del equipo asistencial, competente, efectivo y seguro, honrado y confiable, comprometido con el paciente y con la organización, y que vive los valores del profesionalismo. La Conferencia de Decanos en España, dentro del marco del programa de la ANECA sobre el diseño de planes de estudio y títulos oficiales adaptados al Espacio Europeo de Educación Superior (EEES) y la reforma de Bolonia, adoptó en su modelo formativo las competencias específicas del modelo del Institute for International Medical Education (27), incorporando las competencias genéricas del proyecto Tunning. (Libro blanco de la titulación del grado de medicina, disponible en: www.aneca.es/activin/docs/libroblanco_medicina_def.pdf) 
Respecto a la Psiquiatría, el programa nacional de la especialidad, (BOE $\mathrm{n}^{\circ}$ 224 de 16 de septiembre de 2008, ORDEN SCO/2616/2008, p 37916-21), define con claridad dieciséis competencias que están agrupadas en cinco dominios competenciales: clínico, conocimiento e investigación, comunicación, promoción de la salud, y gestión clínica y ética. Estas competencias son perfectamente agrupables en los dominios competenciales de los modelos expuestos anteriormente.

En Europa la UEMS (Unión Europea de Médicos Especialistas) realizó un estudio para comprobar si los estándares de formación en Psiquiatría establecidos en el año 2003 habían influido en los países miembros de la UE. Los resultados del estudio reflejan una gran heterogeneidad en las prácticas formativas entre los 22 países participantes en el estudio. Cabe destacar la presencia en los programas de una formación teórica sobre la vejez, la intervención social, la comunidad y aspectos relacionados con la ética, los derechos humanos, la etnia, la cultura, el género y la discapacidad. Sin embargo, las competencias relacionadas con el liderazgo, la gestión, la administración, los aspectos económicos, la informática aplicada a la medicina y la telemedicina tienen menor peso (30). En España, los residentes tienen un alto grado de satisfacción con la formación, si bien detectan ciertas deficiencias en relación con la psicoterapia, la metodología de investigación, la gerontopsiquiatría, la neurología y la medicina general (31).

Por otra parte, la formación en las competencias relacionadas con la Ética es objeto de especial tratamiento en la especialidad de Psiquiatría, superior a otras especialidades. En 1996 se editó el documento Declaración de Madrid Sobre los Requisitos Éticos de la Práctica de la Psiquiatría. Sin embargo, algunos autores han detectado una escasa formación en esta competencia y han propuesto recomendaciones para promover la enseñanza de la ética en psiquiatría (32).

Implementación de un programa de competencias, la experiencia de la Unidad de Docencia Médica del Hospital Universitario Cruces.

Es muy importante que las instituciones formadoras (académicas y sanitarias), tomen como punto de partida para definir el perfil profesional, las distintas transiciones sociales. En este análisis deben de participar las organizaciones académicas, sanitarias y profesionales (colegios, sociedades científicas), alumnos y sobre todo escuchar la voz de los ciudadanos, en un trabajo que debe ir más allá de lo local o regional.

Un ejemplo al respecto, es el ya citado proyecto (EFPO-Educating Future Physicians for Ontario) (7), que surgió en Ontario (Canadá) en 1990, tras una huelga de médicos en 1987, donde se puso de manifiesto la gran separación que existía entre la profesión médica y la opinión de la población sobre ésta. A partir de ese 
PARA EL DEBATE

hecho, cinco facultades de medicina de la provincia pusieron en marcha el proyecto EFPO para identificar lo que la población esperaba de sus médicos y proponer modificaciones educativas.

A través de las opiniones de grupos de ciudadanos, expectativas de médicos y otros profesionales y datos sanitarios, se identificaron ocho dominios competenciales del médico: Experto, comunicador, colaborador, defensor de la salud, aprendiz, gestor (guardián de recursos), estudioso y el médico como persona y profesional. La denominación de estas competencias surgió, en la mayor parte de ellas, de expresiones reales de los ciudadanos encuestados. Este proyecto no pretendía solo definir un modelo, sino cambiar la cultura de las instituciones educativas, dando más participación a los ciudadanos. El modelo fue adoptado posteriormente para el conjunto de Canadá bajo las siglas ya conocidas, CanMEDS (8) y ha sido adoptado por Holanda y algunas instituciones formativas de Alemania.

Una vez definido el perfil del profesional, se debe establecer una estrategia de implementación. Un buen ejemplo lo tenemos en el IIME para universidades de China (33), en la formación especializada en Canadá (34), en EEUU (19) y especialmente en la formación especializada en Holanda, con un proyecto dirigido desde el gobierno, realizado por profesionales de la educación médica y desarrollado escalonadamente por los distintos hospitales universitarios (35).

Trabajar con un modelo de este tipo facilita el desarrollo y adaptación del proceso formativo (objetivos de aprendizaje derivados de las competencias, actividades, tareas específicas, itinerarios formativos, cronograma, metodologías y recursos docentes) y realizar una evaluación del desempeño (evaluación por resultados). Para generar el cambio, es muy importante que todos los profesionales trabajen con el mismo planteamiento y lenguaje. Por ello, para que la formación especializada sea un proceso que profesionalice, se precisa: 1) un proyecto común, liderado por la institución y cada responsable del servicio asistencial; 2) una buena gestión de cada programa de formación; 3 ) una buena práctica docente de los tutores; 4) una buena disposición de los residentes para formarse; 5) un buen soporte para la formación (recursos); 6) una colaboración de todos los profesionales, y 7) trabajar todas la competencias con el mismo nivel de importancia (36).

En línea con el marco internacional, la Unidad de Docencia Médica del Hospital Universitario Cruces, basándose en el modelo del IIME, y con incorporaciones de los modelos de Canadá y de EEUU (ACGME), definió en el 2008 las competencias generales del especialista a formar en la llamada "Visión Docente" (37), dentro del proyecto: "Formación Especializada Basada en Competencias (FEBC) en el HU Cruces"(36). El modelo tiene siete dominios competenciales con 32 competencias generales (tabla 4 y figura 2):

1. Valores profesionales, actitudes, comportamiento y ética (profesionalidad).

2. Comunicación.

3. Cuidados del paciente y habilidades clínicas. 
4. Conocimientos médicos. (fundamentos científicos de la medicina).

5. Práctica basada en el contexto del sistema de salud (salud pública y sistemas sanitarios.

6. Práctica basada en el aprendizaje y la mejora continua (análisis crítico y autoaprendizaje).

7. Manejo de la información.

Tabla 4.

Modelo de competencias del hospital universitario cruces 2008.0

Visión Docente: perfil profesional basado en siete dominios competenciales del médico/profesional en el siglo XXI. (ver figura 2)

\begin{tabular}{|c|c|}
\hline $\begin{array}{l}\text { DOMINIO } \\
\text { COMPETENCIAL }\end{array}$ & $\begin{array}{l}\text { COMPETENCIA } \\
\text { EI médico/profesional demostrará que... }\end{array}$ \\
\hline $\begin{array}{l}\text { 1. Valores profesionales, actitu- } \\
\text { des, comportamiento y ética } \\
\text { (Profesionalidad). }\end{array}$ & $\begin{array}{l}\text { a. Proporciona la más alta calidad de cuidados de salud con integridad, honesti- } \\
\text { dad y humanidad, altruismo y sentido del deber, teniendo en cuenta los prin- } \\
\text { cipios éticos (beneficencia, autonomía y justicia) y morales de la profesión y } \\
\text { reconociendo las responsabilidades legales. } \\
\text { b. Toma conciencia de las cuestiones raciales, multiculturales y sociales, y de } \\
\text { la obligación personal de mantener la mejora de los propios conocimientos, } \\
\text { habilidades y conducta profesional. } \\
\text { c. Muestra una adecuada conducta personal e interpersonal en su entorno de } \\
\text { trabajo. } \\
\text { d. Consulta eficazmente con otros colegas y profesionales de la salud. } \\
\text { e. Contribuye eficazmente en las actividades de equipos interdisciplinares (otras } \\
\text { instituciones, comités, grupos de investigación, docencia). } \\
\text { f. Facilita y colabora en el aprendizaje/formación de los pacientes, personal re- } \\
\text { sidente y otros profesionales de la salud } \\
\text { g. Desarrolla, realiza y documenta las estrategias de educación personal y desa- } \\
\text { rrollo profesional continuo. }\end{array}$ \\
\hline 2. Comunicación. & $\begin{array}{l}\text { a. Establece una relación terapéutica con los pacientes y familiares, basada en la } \\
\text { empatía, confianza, comprensión y confidencialidad. } \\
\text { b. Capacidad para obtener y sintetizar la información clínica relevante del pa- } \\
\text { ciente, sus familiares y la comunidad, acerca de los problemas de este. } \\
\text { c. Trata adecuadamente la información con el paciente, su familia y otros pro- } \\
\text { veedores de cuidados de salud para facilitar los cuidados óptimos del pacien- } \\
\text { te. } \\
\text { d. Se comunica adecuadamente con el equipo de trabajo y con su institución. } \\
\text { e. Se comunica adecuadamente con la judicatura y las fuerzas de seguridad en lo } \\
\text { relativo a la protección de la salud individual y colectiva, dentro de los límites } \\
\text { de su responsabilidad. } \\
\text { f. Gestiona la información médica/profesional ante los medios de comunica- } \\
\text { ción, en lo relativo a la información científica y colaboración en la promoción } \\
\text { de la salud, dentro de los límites de su responsabilidad. }\end{array}$ \\
\hline
\end{tabular}


PARA EL DEBATE

\begin{tabular}{|c|c|}
\hline $\begin{array}{l}\text { 3. Cuidados del paciente y habi- } \\
\text { lidades clínicas. }\end{array}$ & $\begin{array}{l}\text { a. Posee conocimientos, habilidades y actitudes en el diagnóstico y tratamiento } \\
\text { para manejar éticamente, con eficacia y eficiencia, los problemas de salud de } \\
\text { los pacientes, dentro de los límites de su especialidad, priorizando la seguri- } \\
\text { dad del paciente y la calidad de los cuidados. } \\
\text { b. Accede y aplica la información relevante para la práctica clínica. } \\
\text { c. Reconoce las limitaciones personales en las actuaciones médicas y colabora y } \\
\text { consulta con otros colegas respecto al cuidado de sus pacientes, su educación } \\
\text { y opiniones sobre temas legales. }\end{array}$ \\
\hline $\begin{array}{l}\text { 4. Conocimientos (fundamentos } \\
\text { científicos de la medicina). }\end{array}$ & $\begin{array}{l}\text { a. Posee sólidos conocimientos de ciencias básicas, clínicas y sociales; entiende } \\
\text { los principios en que se basan las decisiones y los actos médicos, y los aplica } \\
\text { a la solución de los problemas de salud. } \\
\text { b. Toma conciencia de su responsabilidad en el autoaprendizaje y el manteni- } \\
\text { miento de los conocimientos, y se adapta a los cambios demostrando la actua- } \\
\text { lización de los mismos. }\end{array}$ \\
\hline $\begin{array}{l}\text { 5. Práctica basada e el contexto } \\
\text { del Sistema de Salud (Salud } \\
\text { Pública y Sistemas Sanita- } \\
\text { rios). }\end{array}$ & $\begin{array}{l}\text { a. Entiende su responsabilidad en la protección y promoción de la salud en la } \\
\text { comunidad. } \\
\text { b. Identifica los determinantes de salud que afectan al paciente; reconoce y res- } \\
\text { ponde a aquellas circunstancias, objetivos o situaciones en las que es apropia- } \\
\text { do la promoción de la salud (identificando poblaciones a riesgo, proponiendo } \\
\text { políticas de salud, etc.). } \\
\text { c. Comprende los principios de las organizaciones sanitarias, fundamentos eco- } \\
\text { nómicos y legales. } \\
\text { d. Conoce la organización de los centros sanitarios y las funciones del distinto } \\
\text { personal que en ellos trabaja. } \\
\text { e. Distribuye eficaz y eficientemente los recursos dedicados a los cuidados y } \\
\text { educación para la salud; es consciente de sus limitaciones, teniendo en cuenta } \\
\text { la equidad en el acceso a los cuidados y la calidad de los mismos. } \\
\text { f. Utiliza eficazmente el tiempo dedicado al cuidado del paciente y trabaja efi- } \\
\text { caz y eficientemente en las organizaciones sanitarias. } \\
\text { g. Utiliza eficazmente la información tecnológica para optimizar los cuidados } \\
\text { del paciente. }\end{array}$ \\
\hline $\begin{array}{l}\text { 6. Práctica basada en el apren- } \\
\text { dizaje y la mejora continua } \\
\text { (Análisis crítico-Autoapren- } \\
\text { dizaje). }\end{array}$ & $\begin{array}{l}\text { a. Valora críticamente la información científica para aplicarla al cuidado de los } \\
\text { pacientes. } \\
\text { b. Formula hipótesis, recoge y valora críticamente los datos para la solución de } \\
\text { problemas. } \\
\text { c. Razona científicamente y aplica el método científico, comprendiendo el poder } \\
\text { y las limitaciones del mismo cuando se trata de aplicarlo a la causa, el trata- } \\
\text { miento y la prevención de las enfermedades. } \\
\text { d. Entiende la necesidad de la autoevaluación y la evaluación por otros de su } \\
\text { práctica profesional, con el objeto de establecer mejoras de la misma. }\end{array}$ \\
\hline 7. Manejo de la información. & $\begin{array}{l}\text { a. Busca, organiza e interpreta críticamente la información biomédica y de sa- } \\
\text { lud. } \\
\text { b. Comprende las posibilidades y limitaciones de las tecnologías de la informa- } \\
\text { ción. } \\
\text { c. Mantiene los registros adecuados de su práctica clínica a efectos de análisis y } \\
\text { mejora de la misma. }\end{array}$ \\
\hline
\end{tabular}


El objetivo del proyecto es cambiar la cultura de la educación en los residentes, supervisores/tutores y equipos profesionales, a través de nuevos métodos de evaluación basados en los siete dominios competenciales e introducir una modificación en los métodos educativos. El proyecto define la Visión Docente, es decir, el camino a seguir a través de un trabajo coherente en la planificación, desarrollo y evaluación de la formación, con la participación activa de todos los agentes implicados en la formación; tutores, facultativos, personal sanitario y residentes. El fin último es responder al compromiso social derivado de la acreditación docente y a las nuevas necesidades de la sociedad (figura 2).

Figura 2.

Proyecto de FEBC del HU Cruces. Un cambio de la cultura educativa basado en la acreditación como compromiso social y en la Visión Docente.

\section{Visión Docente: \\ Perfil profesional basado en siete dominios competenciales}

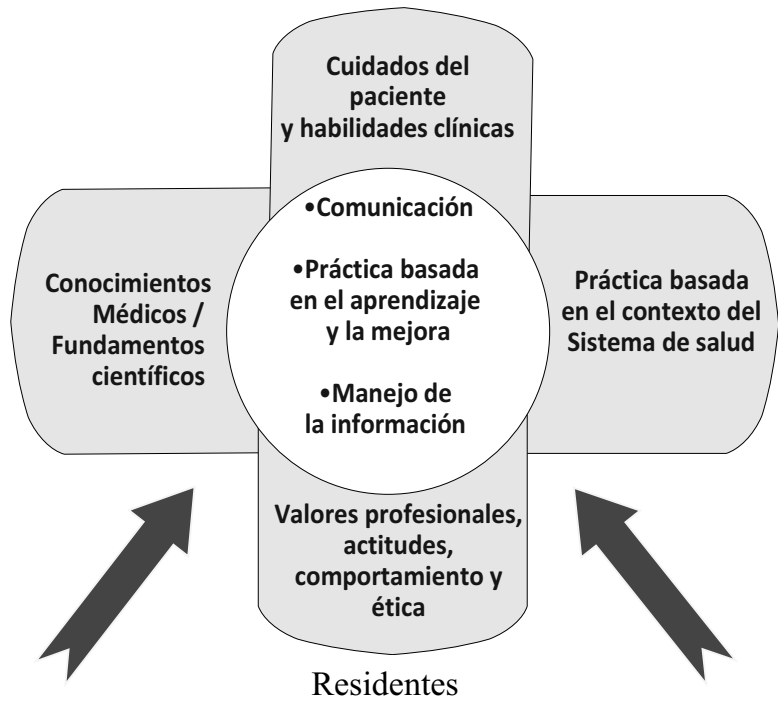

Facultativos

Tutores

Enfermería

Coherencia en la Planificación-Desarrollo-Evaluación de la formación

El compromiso: Acreditación como responsabilidad social 
PARA EL DEBATE

Su desarrollo de 2008 a 2012, se ha basado en tres acciones estratégicas (38):

1. Acciones de Gestión.

a. Aprobación por la Dirección y la Comisión de Docencia. Reuniones formales periódicas con la Dirección, las Jefaturas de los 40 servicios asistenciales, los Tutores e información sistemática a los residentes.

b. Inclusión de la FBC en el Plan Estratégico del hospital.

c. Creación del Comité docente de Jefes de Servicio.

d. Creación de un Comité de Expertos en evaluación de Competencias, formado por tutores y residentes. El objetivo es: diseñar, adaptar, implementar y valorar, los nuevos métodos de evaluación.

2. Formación específica para residentes y tutores en el modelo y en las estrategias de la Formación Basada en Competencias y su significado hoy en la educación.

3. Diseño y aplicación de nuevos métodos de Evaluación formativa basados en los siete dominios competenciales referidos:

a. Formulario de evaluación global (Global Rating Form).

b. Formulario de autoevaluación.

c. Formulario para la entrevista Tutor-Residente (feedback).

d. Portafolio cuantitativo y cualitativo que incluye reflexiones por competencias en los siete dominios.

Tras cuatro años los resultados del proyecto se resumen en: 1) Definición del perfil profesional en la "Visión Docente", 2) el compromiso institucional, 3) el desarrollo mediante una estrategia múltiple a través de una masa crítica de tutores, residentes y jefes de servicio, 4 ) e innovaciones al diseñar y aplicar nuevos métodos de evaluación formativa basados en competencias, y al constituir dos novedosos comités, el comité docente de Jefes de Servicio y el de tutores expertos en evaluación de competencias.

\section{Conclusiones}

1. El perfil del profesional debe responder a las necesidades de la sociedad y del sistema sanitario. Las competencias son dinámicas, al igual que el ritmo de cambio de las transiciones sociales. Es preciso tener en cuenta el concepto de salud y enfermedad de cada cultura. El perfil debe fundamentarse en los principios del profesionalismo, la calidad de los cuidados, la seguridad del paciente y el servicio a la comunidad, y en su definición han de colaborar agentes profesionales, educativos, políticos, gestores, ciudadanos y pacientes.

2. El profesional de hoy debe de tener una formación basada en valores, en principios éticos y en aquellos aspectos que potencien la humanización de la relación con el paciente; disponer de sólidos fundamentos científicos y tecnológicos, y 
de un conocimiento y una práctica del método científico que esté unido a la gestión de la complejidad y de la incertidumbre; manejar correctamente el lenguaje científico, tecnológico e informático para facilitar el aprendizaje autónomo; tener capacidad de iniciativa y trabajo en equipo así como el desarrollo de habilidades para los asuntos personales y para una eficaz participación democrática en la sociedad y en las instituciones sanitarias. En una palabra, es necesario trabajar por la excelencia profesional (39).

3. Las instituciones educativas deberán adaptar sus líneas curriculares, sus metodologías docentes y el perfil de sus educadores, transitando de un modelo educativo exclusivamente centrado en la "enfermedad (órgano y sistema)" a un modelo holístico que además tenga en cuenta el contexto familiar, social y sanitario.

4. La formación ha de incidir en potenciar la convivencia entre las distintas culturas, el liderazgo, una visión universalista, el pensamiento crítico y creativo, la solidaridad y la corresponsabilidad en el sostenimiento de los sistemas sanitarios públicos, máxime en los momentos críticos que se viven actualmente en un mundo interdependiente.

5. La FBC exige de los residentes una autoevaluación, un compromiso explícito con su propio proceso formativo y una reflexión continua documentando los puntos fuertes y débiles. Y por parte del proceso formativo y de evaluación, se requiere un sistema basado en la observación del comportamiento en el puesto de trabajo y de los resultados del trabajo; una retroalimentación frecuente y de alta calidad (feedback) por parte del evaluador; documentar los resultados de aprendizaje y flexibilizar el programa en función del ritmo de aprendizaje. La reflexión es la clave y la evaluación se convierte en el verdadero motor del aprendizaje y del desarrollo profesional siendo el tutor la pieza fundamental de todo este proceso.

6. Numerosas organizaciones han definido el modelo de profesional a formar. Lo importante, es ir más allá de las recomendaciones y ponerlos en marcha modificando las organizaciones educativas y sanitarias y sus culturas. Consideramos que el modelo de FEBC del HU Cruces es útil para todas las especialidades, y está introduciendo la cultura de que todas las competencias tienen el mismo valor a la hora de formar a un residente, resultando ser la primera experiencia en hospitales españoles, según nuestro conocimiento. 
PARA EL DEBATE

\section{BIBLIOGRAFÍA:}

(1) GlaxoSmithKline pagará 2.400 millones de euros por fraude en Estados Unidos. Disponible en: http://sociedad.elpais.com/sociedad/2012/07/03/actualidad/1341267463_727960.html [citado 1 de marzo de 2013]

(2) Morán-Barrios J, Ruiz de Gauna Bahillo P. ¿Reinventar la formación de médicos especialistas?. Principios y retos. Nefrología 2010;30(6):604-12.

(3) ABIM Foundation. American Board of Internal Medicine; ACP-ASIM Foundation. American College of Physicians-American Society of Internal Medicine; European Federation of Internal Medicine. Medical professionalism in the new millennium: a physician charter. Ann Intern Med $2002 \mathrm{Feb}$ 5;136(3):243-6.

(4) Los Fines de la Medicina: El establecimiento de unas nuevas prioridades. (The Goals of Medicine: Setting new priorities). Un proyecto internacional del Hasting Center. Cuadernos de la Fundación Grifols $n^{\circ}$ 11. 2005. Disponible en: http://www.fundaciogrifols.org/collage/images/potter/ tempFiles/potterTmpFile_uuid_28995/cuaderno11.pdf [citado 1 de marzo de 2013]

(5) Frenk J, Chen L, Bhutta ZA, Cohen J, Crisp N, Evans T, Fineberg H, Garcia P,Ke Y, Kelley P, Kistnasamy B, Meleis A, Naylor D, Pablos-Mendez A, Reddy S, Scrimshaw S, Sepulveda J, Serwadda $\mathrm{D}$, Zurayk H. Health professionals for a new century: transforming education to strengthen health systems in an interdependent world. Lancet 2010 Dec 4;376(9756):1923-58.

(6) Declaración de Barcelona de las asociaciones de pacientes, 2003. Disponible en: http:// www.fbjoseplaporte.org/docs/repositori/070806132617.pdf [citado 1 de marzo de 2013]

(7) Neufeld VR, Maudsley RF, Pickering RJ, Turnbull JM, Weston WW, Brown MG, Simpson JC. Educating future physicians for Ontario. Acad Med 1998 Nov;73(11):1133-48.

(8) CanMEDS 2000: Extract from the CanMEDS 2000 Project Societal Needs Working Group Report. Med Teach 2000;22(6):549-54.

(9) Jovell A. El futuro de la profesión médica. Fundació Biblioteca Josep Laporte 2001. Disponible en: http://www.fbjoseplaporte.org/docs/repositori/070517121543.pdf [citado 1 de marzo de 2013]

(10) Oriol-Bosch A. Ser Médico, hoy. Retos del nuevo profesionalismo médico en España. Informe de la Fundación Educación Médica para el Consejo General de Colegios Oficiales de Médicos. Madrid 2006. Disponible en: http://www.educacionmedica.net/sec/serMedico 2006.pdf [citado 1 de marzo de 2013]

(11) Martínez-Clares P, Martínez-Juarez M, y Muñoz-Cantero JM Formación basada en competencias en educación sanitaria: aproximaciones a enfoques y modelos de competencia. RELIEVE, 2008; 14 (2):1-23. (www.uv.es/RELIEVE) [citado 1 de marzo de 2013]

(12) Frank JR, Snell LS, Cate OT, Holmboe ES, Carraccio C, Swing SR, et al. Competencybased medical education: theory to practice. Med Teach 2010;32(8):638-45.

(13) Bunk GP. Teaching Competente in Initial and Continuing Vocational Training in the Federal Republic of Germany. Vocat Train Eur J 1994;1:8-14.

(14) Echeverría B. Gestión de la competencia de Acción Profesional. Revista de Investigación Educativa 2002; 20 (1): 7-43.

(15) McClelland D. Testing for Competence rather than for Intelligence. Am Psychol 1973;28(1):1-14.

(16) Holmboe ES, Sherbino J, Long DM, Swing SR, Frank JR. The role of assessment in competency-based medical education. Med Teach 2010;32(8):676-82. 
(17) Iobst WF, Sherbino J, Cate OT, Richardson DL, Dath D, Swing SR, et al, for The International CBME Collaborators. Competency-based medical education in postgraduate medical education. Med Teach 2010; 32: 651-6.

(18) Roma Millán J.La reflexión como eje central del desarrollo profesional.Educ Méd 2007;10(1):306. Disponible en: http://scielo.isciii.es/pdf/edu/v10n1/formacion.pdf. [citado 1 de marzo de 2013]

(19) Schuwirth L W T and Van der Vleuten C P M. Programmatic assessment: From assessment of learning to assessment for learning. Med Teach 2011; 33 (6): 478-85.

(20) Kogan JR, Holmboe ES, Hauer KE. Tools for Direct Observation and Assessment of Clinical Skills of Medical Trainees. A systematic review. JAMA 2009;302(12):1316-26.

(21) Epstein RM. Assessment in Medical Education. N Engl J Med 2007;356:387-96.

(22) Norcini J, Burch V. Workplace-based assessment as an educational tool: AMEE Guide No. 31. Med Teach 2007;29(9-10):855-71.

(23) Joint Royal Colleges of Physicians Trainning Board. Assessment. Workplace-Based Assessment Documents. Disponible en: http://www.jrcptb.org.uk/assessment/Pages/WPBA-Documents. aspx [citado 1 de marzo de 2013]

(24) Lelliott P, Williams R, Mears A, Andiappan M, Owen H, Reading P, Coyle N, Hunter S. Questionnaires for 360-degree assessment of consultant psychiatrists: development and psychometric properties. Br J Psychiatry 2008 Aug;193(2):156-60.

(25) Scottish Deans' Medical Curriculum Group. Learning Outcomes for the Medical Undergraduate in Scotland: A Foundation for Competent and Reflective Practitioners. 3rd Edition. August 2007. Disponible en: www.scottishdoctor.org/resources/scottishdoctor3.doc [citado 1 de marzo de 2013]

(26) Swing SR. The ACGME outcome project: retrospective and prospective. Med Teach 2007 Sep;29(7):648-54.

(27) Core Committee, Institute for International Medical Education. Global minimum essential requirements in medical education. Med Teach. 2002 Mar;24(2):130-5. Traducción al español: Requisitos Globales Mínimos Esenciales en Educación Médica. Educ. Méd [online]. 2003:6 (2): 11-9. Disponible en: http://scielo.isciii.es/scielo.php?script=sci_arttext\&pid=S1575-18132003000400003\&lng=es\&nrm=iso

(28) Ladhani Z, Scherpbier AJ, Stevens FC. Competencies for undergraduate community-based education for the health professions. A systematic review. Med Teach 2012;34(9):733-43.

(29) Pardell H y Fundación Educación Médica. El médico del futuro. Disponible en: http://www. educmed.net/sec/serMédico2009.pdf. [citado 1 de marzo de 2013]

(30) Lotz-Rambaldi W, Schäfer I, ten Doesschate R, Hohagen F. Specialist training in psychiatry in Europe--results of the UEMS-survey. Eur Psychiatry 2008 Apr;23(3):157-68.

(31) Gómez Beneyto, Manuel; Montilla-García, José F.; Castro Manglano, P. de. La opinión de los residentes de psiquiatría sobre la formación que reciben. Actas Esp Psiquiatr 2011, 39(3): 174-79.

(32) Bloch S, Green SA. Promoting the teaching of psychiatric ethics. Acad Psychiatry 2009 Mar-Apr;33(2):89-92.

(33) Schwarz MR, Wojtczak A, Stern D. The outcomes of global minimum essential requirements (GMER) pilot implementation in China. Med Teach 2007; Sep;29(7):699-705.

(34) Frank JR, Danoff D. The CanMEDS initiative: implementing an outcomes-based framework of physician competencies. Med Teach 2007 Sep;29(7):642-7. 
PARA EL DEBATE

(35) Scheele F, Teunissen P, Van Luijk S, Heineman E, Fluit L, Mulder H, Meininger A, WijnenMeijer M, Glas G, Sluiter H, Hummel T. Introducing competency-based postgraduate medical education in the Netherlands. Med Teach 2008;30(3):248-53.

(36) Morán-Barrios J. La Evaluación del proceso de Formación Especializada. Una propuesta fundamentada en el proyecto de "Formación Especializada Basada en Competencias del Hospital de Cruces 2008”. Educ Méd 2012; 15 (supl 1): S29-S34. Disponible en: http://www.educmed.net/pdf/ revista/15S01/2512058_AAVV_mdp_EDU.pdf [citado 1 de marzo de 2013]

(37) Morán-Barrios J. Ser Médico/Profesional. Disponible en: http://extranet.hospitalcruces. com/doc/adjuntos/Vision\%20docente-ser\%20medico\%202012.pdf. [citado 1 de marzo de 2013]

(38) Morán-Barrios J, Somme J, Iglesias G, Amutio E, Martínez-Berriotxoa A, Saá R Development of "Competency-based Postgraduate Training (CBPT) Cruces Hospital Project-2008" (2008-2011). The first experience in Spanish hospitals. Proceedings of AMEE 2012. Disponible en: http:// www.amee.org/documents/AMEE\%202012\%20Abstract\%20Book\%20Updated.pdf [citado 1 de marzo de 2013]

(39) Camps V. La excelencia en las profesiones sanitarias. Fundación Medicina y Humanidades Médicas. Disponible en: http://www.fundacionmhm.org/tema0721/revista.html [citado 1 de marzo de 2013] 